\title{
INFLUENCE OF THE SCLERAL LENS AND FLUID RESERVOIR THICKNESS ON RESIDUAL ASTIGMATISM
}

Langis Michaud OD, MSC, FAAO (Dipl)

Gabriella Courey OD

Ecole d'optométrie de L'Université de Montréal

Corresponding author: langis.michaud@umontreal.ca

Submitted: June 25, 2020. Accepted: January 7, 2021. Published: January 14, 2021.

\section{ABSTRACT}

\section{Purpose}

This study aims to determine if lens or tear fluid reservoir thicknesses (LT/FRT) may influence the presence of residual astigmatism and participant's visual acuity.

\section{Methods}

The study was a randomized, non-dispensing, prospective study. Empirically and randomly chosen participants were fitted with 4 combinations ( 350 and 250 um LT fitted with 250 and 350 um FRT) of 16 mm diameter scleral lenses, designed using a corneo-scleral profiler software (sMap 3D, Visionary Optics, US). Lenses haptics were kept spherical for all lenses. They were evaluated under a slit lamp, anterior segement OCT (objective fluid reservoir and lens thicknesses), topography over lenses and aberrometry, after 30 minutes of lens wear. Spherico-cylindrical refraction and logMar acuity were also assessed.

\section{Results}

Study population was composed of 24 participants aged $24.2+4.7$ years old. Baseline refractive error was $-2.3+1.6 \mathrm{D}$ with $-0.48 \pm 0.26 \mathrm{D}$ of astigmatism. In vivo (OCT) lens A was $344.1 \pm 15.4$ um thick, fitted with a vault of $213.6 \pm 42.4 \mathrm{um}$; Lens $\mathrm{B}$ was $346.2 \pm 12.5 / 327.2 \pm 44.8$; Lens $\mathrm{C}$ was $260.3 \pm 17.7 / 214.0 \pm$ 40.6 um and Lens $\mathrm{D}$ was $262.2 \pm 13.2 / 330.8 \pm 52.0$ respectively. All lenses were found similarly decentered inferiorly by 0.10 to $0.15 \mathrm{um}$. BCVA was $-0.32+0.08$ (A), $-0.21+0.10(\mathrm{~B}),-0.28+0.08$ (C), and -0.14 +0.10 (D), compared to $-0.25+0.08$ (A), $-0.11+0.10$ (B), $-0.23+0.06$ (C), and $-0.05+0.12$ (D) when sphere only was compensated. Residual refractive astigmatism $(\mathrm{RA}=-0.50$ to $-0.75 \mathrm{D})$ is found significantly higher based on the FRT ( $\mathrm{F}=9.560 ; \mathrm{p}=0.037)$ and not $\mathrm{LT}(\mathrm{F}=0.429 ; \mathrm{p}=0.522)$. There is no correlation between RA and over-k readings (Lens A r $=-0.078, \mathrm{p}=0.773$; Lens B $\mathrm{r}=-0.073$, $\mathrm{p}=0.788$; Lens $\mathrm{C} \mathrm{r}=-0.345$, $\mathrm{p}=0.171$; Lend $\mathrm{D} r=0.019, \mathrm{p}=0.944)$. Higher order aberrations, mostly vertical coma, were found clinically 
significant but not statistically different between lenses $(A=0.350+0.032 ; B=0.382+0.053, C=0.329+$ 0.044 and $\mathrm{D}=0.385+0.062 ; \mathrm{p}=0.776$ )

\section{Conclusion}

This study proves that low level of RA may be found when scleral lenses are fitted on normal corneas. Its occurrence is related to the presence of high-order aberrations and less likely to lens flexure.

Key Words: scleral lenses, residual astigmatism, lens flexure, high-order aberrations

\section{INTRODUCTION}

Scleral lenses (SL) are large rigid devices that are increasing in popularity. These lenses are fitted to vault over the cornea and to land on the non-sensitive and smooth conjunctiva. Indications for SLs include vision improvement in adult and pediatric patients with corneal ectasia, ocular surface protection, and medical issues in situations where standard treatment is not possible or ineffective. ${ }^{1}$ They are also becoming a valid option for healthy corneas when other contact lens modalities fail to provide the desired comfort. ${ }^{2}$

In general, SLs also make it possible to improve patients' visual acuity, mainly through the optical impact of the fluid reservoir, which helps compensate for the cornea's surface irregularities. However, there are still several cases where visual acuity remains below expected levels, despite sphero-cylindrical over-refraction. One of the often-cited reasons to explain this outcome relates to the lens flexure on the ocular surface.

Flexure is known as a gas-permeable (GP) corneal lens's tendency to bend when it is worn. It is detected by over-keratometry ${ }^{3}$ and is well documented in corneal gas-permeable lenses. ${ }^{4}$ It is recognized as a factor that may alter the optical correction of astigmatism, wanted or not, especially when the curves of the GP lens do not conform with the corneal shape. In order to flex, GP lens must be made thin $(<0.13 \mathrm{um}),{ }^{5}$ and is influenced by the nature of the material and the corneal toricity. Flexure also increases as the tear layer under the lens is thicker. Finally, lid tension and tear viscosity can also play a role.

The flexure phenomenon was less investigated with larger and thicker SL. Residual astigmatism (RA) found when wearing SL was first associated with lens flexure, as suggested by over-keratometry findings. ${ }^{6}$ A more recent prospective study ${ }^{7}$ demonstrated that lens flexure may exist and be influenced by the lens thickness but failed to correlate flexure and RA. This confirms the author's personal clinical experience that increasing lens thickness does not improve visual acuity when RA is found.

It has been suggested that several elements can influence the refractive correction of the eye through SLs. The most important are related to the lens itself (power, base curve), then those related to the cornea (steep keratometric reading). Finally, it was hypothesized that the reservoir thickness ${ }^{8}$ may play a role, but it was not proven so far. ${ }^{9}$

More recently, residual astigmatism (RA) was found as a result of non-compensated internal optics of the eye or as a result of the lens decentration ${ }^{10}$ and its consequent fluid reservoir prismatic shape. ${ }^{11}$ Reduced visual acuity when wearing SLs was also associated with the presence of non-corrected high-order aberrations, especially in the case of corneal ectasia. ${ }^{12}$

It may be difficult to determine exactly which parameters influence the most the optical outcome when wearing SLs.

\section{OBJECTIVE}

To limit the number of factors involved, this study was made on a normal cornea population, with no excessive steep corneal curvature and no physiological astigmatism. Factors related to the lenses were also kept constant for the most, except for the lens and reservoir thicknesses. Consequently, this study aims to determine if these parameters may influence the presence of residual astigmatism and the participant's visual acuity.

\section{METHODS}

This prospective study was randomized and nondispensing. The investigational review board approved

J Cont Lens Res Sci Vol 5(1):e1-e8; January 14, 2021

This article is distributed under the terms of the Creative Commons Attribution-Non Commercial 4.0 International License. @Michaud and Courey. 
the study at Université de Montréal. All procedures complied with the tenets of the Declaration of Helsinki, as revised in 2000.

The first 24 patients meeting the criteria listed in Table 1, and has provided informed consent, were enrolled. Once participants were found eligible, their ocular surface was assessed and 4 SLs were designed, based on a corneo-scleral profiler data (sMap 3D, Visionary Optics, US) to be thick or thin, and designed to vault with low or high reservoir thickness. Specifically, this means: a lens with a standard central thickness (CT) of 350 microns (um), designed to be fitted with 200 um fluid reservoir thickness (FRT) (Lens A); or 350 um FRT (Lens B), a lens with CT of 250 um- fitted with 200 um FRT (Lens C) or 350 um (lens D)As recommended by the manufacturer. The lens's power was determined after trial of a smaller GP lens, of a known base curve, and over-refraction with this equipment on the eye. These data (GP lens base curve and final power) were computed in the manufacturer's software to design the final scleral lens parameters. All lenses were manufactured with a diameter of 16 $\mathrm{mm}$ and spherical haptics. The material selected was Hexafocon A (Polymer Technology, US). All lenses were ordered and provided by the same manufacturer (Visionary Optics, US).

When the manufacturer delivered lenses, they were measured for power (Topcon lensometer) and lens thicknesses using a manual lens thickness gauge (W0-600, Western Opticals). CT was determined at the lens's geometric center, and nasal and temporal thicknesses were also evaluated at $6 \mathrm{~mm}$ from the central point, along the same meridian. Data are reported in Table 2.

Lenses A and B were randomly selected to be applied to the participant's eye (OD or OS) in the first session. At least $48 \mathrm{~h} 00$ later, corresponding lenses (C
TABLE 1 Inclusion and Exclusion Criteria Inclusion criteria

1. Must be between 18 and 40 years of age.

2. Must have a refractive error between $-0.25 \mathrm{D}$ and $-6.00 \mathrm{D}$ and with less than $1.00 \mathrm{D}$ of corneal cylinder.

3. Must present normal ocular health.

4. Must not have worn contact lenses for the past 48 hours.

5. Must be legally able to consent to participate in this study.

6. Must be available for 3 visits in non-consecutive days.

\section{Exclusion criteria}

1. Must not have an abnormal ocular surface (including corneal ectasia).

2. Must not have measurable lenticular astigmatism.

3. Must not have worn contact lenses for extended wear (part-time or full-time) over the last 6 months.

4. Must not have an active ocular infection at the moment of the clinical trials.

5. Must not be currently using topical medication.

6. Must not have a known hypersensitivity or allergy to the products used during this trial.

7. Must not be a gas-permeable lens wearer, small or sclerals.

vs A and D vs B) were applied the same way during a second session. At every visit, lenses were filled with unpreserved saline solution $(0.9 \% \mathrm{NaCl})$. The lens's evaluation was made 30 minutes post-application, under slit lamp, to evaluate lens alignment and the absence of touch or presence of a bubble. Anterior segment OCT (Optovue iVue SD-OCT, Clarion) was performed to assess central FRT. Three images were taken at the center of the pupil and the tear reservoir thickness was averaged (mean of 3 lectures). A single, trained and experienced technician evaluated FRT using built-in calipers. Subjective sphero-cylindrical

TABLE 2 Baseline Parameters

\begin{tabular}{|l|c|c|c|c|}
\hline & \multicolumn{2}{|c|}{ OD } & \multicolumn{2}{c|}{ OS } \\
\hline & Range & Average & Range & Average \\
\hline Refractive error - sphere (D) & -0.50 to -4.75 & $-2.25+1.62$ & -0.75 to -5.00 & $-2.75+1.87$ \\
\hline Refractive error- astigmatism (D) & -0.25 to -0.75 & $-0.50+0.37$ & 0.00 to -0.75 & $-0.42+0.21$ \\
\hline K - steep & $43.37-44.50$ & $43.87+0.75$ & $43.00-44.62$ & $43.62+0.80$ \\
\hline K - flat & $42.75-43.87$ & $43.12+0.60$ & $42.50-43.12$ & $43.00+0.40$ \\
\hline
\end{tabular}

J Cont Lens Res Sci Vol 5(1):e1-e8; January 14, 2021

This article is distributed under the terms of the Creative Commons Attribution-Non Commercial 4.0 International License. (M) Michaud and Courey. 
over-refraction was also completed. Best-corrected visual acuity at a distance was noted (logMar) using ETDRS chart, calibrated at 6 meters, under photopic illumination. According to Vincent's technique, ${ }^{7} 10$ topographical maps (Medmont, Precision Ophtalmics) over the lenses were taken and averaged to assess lens flexure, at $12 \mathrm{~mm}$ chord value. Finally, aberrometry was performed when lenses were worn. Results were evaluated for a $4 \mathrm{~mm}$ pupil size (the smaller found within participants).

\section{STATISTICAL ANALYSIS}

After coding, the data have been entered to an Excel Sheet and exported to, processed, and analyzed using SPSS version 25. Descriptive statistics such as mean and standard deviation (SD) were used to summarize the descriptive data. An ANOVA for repeated measurements was used to analyze the relationship between cylindrical and spherical equivalent over-refraction and over-keratometry findings and FRT or lens thickness for each lens tested. If no significant interaction was found, a variation of the parameters were estimated with a confidence interval at $95 \%$ level.

\section{RESULTS}

The participants' cohort was composed of 14 females and 10 males, aged $24.2 \pm 4.7$ years old. All were diagnosed with normal cornea based on slit lamp and topographic mapping. Baseline refractive and corneal parameters are detailed in Table 2 Bestcorrected visual acuity was $-0.13 \pm 0.10$ OD and $-0.08 \pm 0.13$ OS.

All lenses were empirically designed and all carried spherical peripheral curves. Table 3 presents the results for each lens tested. Empirical calculation from the software was inefficient, and most lenses aiming to vault with higher FRT came off power.

Manufacturing was also an issue. On average, lenses A and B were 5.9 um thinner (-1.6\%) compared to the lens ordered. To the contrary, lenses $\mathrm{C}$ and $\mathrm{D}$ were made thicker by 10 microns on average than ordered, $(+4 \%)$. Nonetheless, the difference in lens thickness between lenses A-B and C-D is 80 um (vs 100 um targeted), which may be considered sufficient to demonstrate if this lens parameter influences the presence of residual astigmatism.

TABLE 3 Results for Each Lens Tested

\begin{tabular}{|l|c|c|c|c|}
\hline & LENS A & LENS B & LENS C & LENS D \\
\hline Lens Power (D) & $-2.4 \pm 2.2$ & $-2.9 \pm 1.9$ & $-1.7 \pm 1.8$ & $-2.1 \pm 0.9$ \\
\hline Central lens Thickness (um) & $344.1 \pm 15.4$ & $346.2 \pm 12.5$ & $260.3 \pm 17.7$ & $262.2 \pm 13.2$ \\
\hline $\begin{array}{l}\text { Nasal lens thickness (um) } \\
@ \text { 6 mm }\end{array}$ & $366.4 \pm 11.8$ & $362.1 \pm 13.7$ & $285.3 \pm 19.5$ & $289.6 \pm 15.8$ \\
\hline $\begin{array}{l}\text { Temporal lens thickness } \\
\text { (um) @ 6 mm }\end{array}$ & $362.1 \pm 13.4$ & $360.7 \pm 11.3$ & $288.9 \pm 17.5$ & $288.4 \pm 16.2$ \\
\hline FRT (um) & $213.6 \pm 42.4$ & $327.2 \pm 44.8$ & $214.0 \pm 40.6$ & $330.8 \pm 52.0$ \\
\hline Over-Ref - sphere (D) & $-0.1 \pm 0.8$ & $-1.2 \pm 2.6$ & $-0.3 \pm 1.6$ & $-1.1 \pm 2.9$ \\
\hline Over-Ref - cyl (D) & $-0.5 \pm 0.3$ & $-0.7 \pm 0.3$ & $-0.5 \pm 0.3$ & $-0.7 \pm 0.2$ \\
\hline Over-Ref - Sph. Equ. (D) & $-0.5 \pm 1.4$ & $-1.5 \pm 2.6$ & $-0.5 \pm 1.5$ & $-1.3 \pm 2.9$ \\
\hline Over-K (D) & $0.2 \pm 0.2$ & $0.3 \pm 0.1$ & $0.3 \pm 0.1$ & $0.3 \pm 0.2$ \\
\hline $\begin{array}{l}\text { VA with sphere corrected } \\
\text { (logMAr) }\end{array}$ & $-0.25 \pm 0.08$ & $-0.11 \pm 0.10$ & $-0.23 \pm 0.06$ & $-0.05 \pm 0.12$ \\
\hline $\begin{array}{l}\text { VA with sphere and cylinder } \\
\text { corrected }\end{array}$ & $-0.32 \pm 0.08$ & $-0.21 \pm 0.10$ & $-0.28 \pm 0.08$ & $-0.14 \pm 0.10$ \\
\hline $\begin{array}{l}\text { Inferior Lens decentration } \\
\text { (mm) }\end{array}$ & $0.15 \pm 0.12$ & $0.12 \pm 0.18$ & $0.10 \pm 0.08$ & $0.14 \pm 0.19$ \\
\hline $\begin{array}{l}\text { High-order aberrations } \\
\text { (total) }\end{array}$ & $0.350 \pm 0.032$ & $0.382 \pm 0.053$ & $0.329 \pm 0.044$ & $0.385 \pm 0.062$ \\
\hline \begin{tabular}{l} 
Coma \\
\hline
\end{tabular} & $0.184 \pm 0.020$ & $0.201 \pm 0.036$ & $0.194 \pm 0.019$ & $0.173 \pm 0.041$ \\
\hline
\end{tabular}


Planned FRT was supposed to reach 200 and 350 um for thin and thick reservoirs, respectively. After lens stabilization (30 minutes), the OCT measurement was 213 and 330 um on average, representing +13 and -20 um of difference from the target. The shallower reservoir, considering SD of 40 um, may represent the condition observed with scleral lens wearers, at the end of the day, when FRT is expected to lie around 200-250 um. ${ }^{12}$ The thicker reservoir, around $350 \mathrm{um}$, is representative of the maximal initial FRT after lens 30 minutes stabilization after application, ${ }^{13}$ assuming that FRT loss during the entire day is $75 \mathrm{um}$ in average, ${ }^{14}$ regardless of the fluid used ${ }^{15}$ The goal of comparing thick and thin reservoirs is kept.

Lens power was slightly more myopic for lenses fitted with higher FRT. This can be explained by the fact that the power was not appropriately compensated for using a steeper base curve to generate a higher vault. Despite this deceiving element, over-refraction allowed reaching optimal visual acuity in all cases. Spherical equivalent over-refractive power was -0.523 for lenses fitted with shallower reservoir, compared to -2.42 for those fitted with high FRT (difference $=$ 1.899; 95\% CI 0.526-3.272).

Residual refractive astigmatism is found significantly higher based on the FRT $(\mathrm{F}=9.560 ; \mathrm{p}=0.037)$ and not the lens thickness $(\mathrm{F}=0.429 ; \mathrm{p}=0.522)$, neither the combined effect of FRT*lens thickness $(\mathrm{F}=0.155$; $\mathrm{p}=0.890)$. Lenses fitted with higher FRT generate more residual astigmatism than those with shallower reservoir (difference $=0.203 ; 95 \%$ CI $0.063-0.343$ ) . This does not influence visual acuity; all participants seeing better than 20/20, regardless of the lens used. VA corrected for the sphere only is slightly lower than if astigmatism is fully compensated, but this is not statistically nor clinically significant.

Considering over-keratometry values, from topography, there was no significant difference based on the FRT $(\mathrm{F}=1.846 ; \mathrm{p}>0.05)$, the lens thickness $(\mathrm{F}=0.007 ; \mathrm{p}>0.890)$ or a combined effect of these 2 elements $(\mathrm{F}=1.556 ; \mathrm{p}=0.432)$. Statistical analysis established that there is no correlation between RA and over-keratometry readings (Lens A $\mathrm{r}=-0.078$, $\mathrm{p}=0.773$; Lens $\mathrm{B} r=-0.073, \mathrm{p}=0.788$; Lens $\mathrm{C} \mathrm{r}=-0.345$, $\mathrm{p}=0.171 ;$ Lend $\mathrm{D} r=0.019, \mathrm{p}=0.944$ )
Finally, high-order aberrations did not vary between lenses $(\mathrm{F}=0.084 ; \mathrm{p}=0.776)$, and their level was considered clinically significant $(>0.300 \mathrm{um})$, which means that patients can report symptoms related to their presence. Coma was identified as the most prevalent aberration with every lens tested. This factor is not influenced by lens centration, all of them stabilizing inferiorly with no significant differences between them.

\section{DISCUSSION}

In general, our results indicate that residual astigmatism is present at a low level $(-0.50 \mathrm{D}$ to $-0.75 \mathrm{D})$ with limited impact on visual acuity. The study aimed to determine if this type of RA would be influenced by lens or TFR thicknesses. Results indicate that RA is influenced by the fluid reservoir thickness independently of the lens thickness. This is an important finding, aligned with previous work by Vincent et al, ${ }^{7}$ who did not find any relationship between RA and lens thickness. This does not seem to be related to lens flexure or torsion, over-keratometry values being not correlated with refractive residual astigmatism findings regardless of the lens or fitting parameters.

Lens flexure is a known phenomenon in GP lens fitting. ${ }^{16}$ Many factors can explain its occurrence but one study may help to understand better the behavior of SLs on the eye. Corzine and Klein found that flexure occurs mostly when lenses are fitted steeper than the corneal curvature, or when space exists between the lens and the eye, and, this is the important point, when this space is not filled appropriately by the tear film. ${ }^{5}$ If SLs are fitted with a vault over the corneal surface, they are also fitted with a reservoir full of fluid. This fluid layer not only compensates for corneal irregularity but also contributes to support the lens mass and to distribute its weight. Consequently, chances to generate flexure is minimal, except when scleral toricity is high. ${ }^{7}$ Moreover, in the case of SLs, flexure is limited, knowing that any lens thicker than $0.25 \mathrm{~mm}$ is considered not flexible. ${ }^{17}$ Other authors may argue that lid tension may cause lens bending because it is supported by spongy tissue (conjunctiva). Consequently, during the blink, lens is pushed deeper in the conjunctival tissue and is expected to bounce back when the lid pressure is removed. This is a theoretical concept that was never proven. It is also hard

J Cont Lens Res Sci Vol 5(1):e1-e8; January 14, 2021

This article is distributed under the terms of the Creative Commons Attribution-Non Commercial 4.0 International License. (M) Michaud and Courey. 
to believe that such lens movement will occur after the full lens stabilization on the eye. Another point to consider is the lens alignment with the conjunctiva. In this study, spherical haptics were used when fitting $16 \mathrm{~mm}$ lenses. It is known that conjunctiva is toric in most cases and that this toricity increases as we progress away from the limbus. A spherical periphery, not well aligned, may lead to increase lens instability and then may contribute to generating more flexure. This was not the case based on our results.

Another critical question to raise is the way we evaluate the lens flexure while on the eye. The most current approach is to take an over-keratometry or to conduct a topography over the lens on the eye. ${ }^{18} \mathrm{We}$ have to remember that keratometry and topography are based on the reflection of mires on the corneal surface, which depends on the quality of the tear film. Keratometry and topography evaluate the structure through a tear film mimicking its shape. Consequently, wetting and re-wetting during the act of blinking may be the cause of the "curvature" variation that we attribute to lens flexure. Topography or keratometry are also static measurements while flexure is considered occurring during blinking, then under a dynamic process. It is hard to believe that a static measurement may properly evaluate a dynamic process occurring when the eye is blinking.

High order aberrations have to be taken into account to appreciate over-refraction results. A scleral lens helps to mask anterior corneal irregularities, in part, through refractive index variation of the fluid reservoir and the corneal surface. These indices are not perfectly matching, and consequently the interface generates anterior corneal surface aberrations. ${ }^{19}$ These aberrations are added to those coming from the irregular posterior cornea, polluting the visual acuity provided through the GP lens surface. ${ }^{20}$ Keratoconus patients experience then the perception of glare and halos, shadows around the letters, and stretching of the images. These optical symptoms were associated, in the past, with astigmatism while in fact, they are the consequence of high levels of horizontal or vertical coma, the latter is recognized as one of the best indicators of the sub-clinical keratoconus. ${ }^{21}$

The results of this study confirm the presence of high-order aberrations, which are more pronounced when the TFR is higher. This difference is not statistically significant. Based on the fact the study was made on a population with normal corneas, such aberrations cannot be generated by the cornea. Their presence may be explained by lens decentration, especially if peripheral curves are not toric. ${ }^{22}$ In fact, decentered lenses can generate a lot of high-order aberrations, particularly comas. ${ }^{23}$ However, results indicate that all lenses behave the same. Decentration is consequently not in play. Remains the change of indices between lens, fluid and corneal surface where lens/TFR thickness may play a role. This is suggested by the fact that thicker TFR are associated with an increased level of HOAs. Among them, vertical coma was the most pronounced.

Optically, a coma is generated when different zones of the lens image rays from an off-axis object point. The image on the retina then lies partly in front and partly behind the fovea. The usual way to reduce coma is to employ a diaphragm to eliminate the outer cones of rays. For more versatile correction of coma, one can use a combination of 2 lenses, both of which are corrected for zero coma at infinite object distance. The appropriate separation of these 2 lenses can correct for coma at various object distances. ${ }^{24}$ Toric lenses are not optically able to compensate for coma, then front-toric SL cannot improve visual acuity when high HOAs are present. It needs SL with a front surface designed to compensate high-order aberrations, ${ }^{12}$ to fix this issue, but, unfortunately, this type of lens is not widely available.

Finally, some manufacturers would suggest increasing the lens thickness to compensate for residual astigmatism. Thicker lenses in this study developed as much astigmatism compared to thinner lenses on the same eye. Consequently, it may not be considered as a valid option to fix refractive issues.

Several factors limit this study. The methods would have been better to test the same eye with the 4 combinations of lenses generated. It was not possible to do so because of limited resources to conduct the study and the time to complete data acquisition. It would had been difficult to recruit participants to come 4 times, every 2 to 3 days. The other limitation is obviously the way lenses were designed to make the process as precise and objective as possible. Specific

J Cont Lens Res Sci Vol 5(1):e1-e8; January 14, 2021

This article is distributed under the terms of the Creative Commons Attribution-Non Commercial 4.0 International License. CMichaud and Courey. 
vault values were needed, so it was decided to use software to design lenses after optical mapping of the ocular surface. This process is time-consuming, and data acquisition may not be as accurate as it should be. This process may not be as convenient as it is supposed to be. If the vault and the power of some lenses were accurate, those needing to vault more over the ocular surface were not well calculated through this method. Lenses produced were also slightly off for the targeted thickness and obviously for the power in a fair number of lenses. This situation may have influenced results presented here. The software used was an early version, but it is known that the manufacturer improved it since this study. Finally, our result apply only on the lens design tested and to a population of normal cornea young adults as described here.

\section{CONCLUSION}

This study suggests that limited residual astigmatism may be found when SLs are fitted on normal corneas. Its occurrence is likely related to the presence of high-order aberrations and not entirely to lens flexure. The amount of RA is influenced by the vault (fluid reservoir) but not the lens thickness. There is no correlation between over-keratometry over the lens and RA found. This means that flexure is most likely not the cause of this residual astigmatism.

\section{ACKNOWLEDGMENTS AND DISCLOSURE}

Lenses were provided by Visionary Optics.

Dr Sheila Morrison, OD for helping with data acquisition.

No other funding was received.

\section{REFERENCES}

1. Johns L. and Barnett M. Contemporary Scleral Lenses: Theory and Application. Bentham eBooks; 2017.

2. van der Worp E, et al. Modern scleral contact lenses: A review. Cont Lens Anterior Eye 2014;37(4):240-50.

3. Goldberg JB. RGP contact lens adherence: Flexure or tear film thinning - Can we define the cause? Internat Contact Lens Clinic 1994;21(1):26-29.

4. Lin MC and Snyder C. Flexure and residual flexure with RGP lenses. Contact Lens Ant Eye 1999;26(1):5-9.
5. Corzine JC and Klein SA. Factors determining rigid contact lens flexure. Optom Vis Sci 1997;74(8): $639-45$.

6. Jedlicka J. Solving scleral lens complications. C.L. Spectrum 2012; 27(October):40-44.

7. Vincent SJ, et al. The influence of centre thickness on miniscleral lens flexure. Cont Lens Anterior Eye 2019;42(1):63-69.

8. Schornack M, Nau C, and Brown W. Estimation of refractive power of scleral lens/fluid reservoir optical systems in non-parallel scleral lens fits. in Poster presented at: Global Specialty Lens Symposium 2014.

9. Bray C, et al., Change in over-refraction after scleral lens settling on average corneas. Ophthalmic Physiol Opt 2017; 37(4):467-72.

10. Ramdass S, Rosen C, and Norman C. Clinical Analysis of Scleral Lenses on Regular Cornea, in Global Specialty Lens Symposium. 2016: Las Vegas.

11. Vincent SJ and Fadel D. Optical considerations for scleral contact lenses: A review. Cont Lens Anterior Eye 2019.

12. Hastings GD, et al. Comparison of wavefront-guided and best conventional scleral lenses after habituation in eyes with corneal ectasia. Optom Vis Sci 2019;96(4):238-47.

13. Michaud L, et al. Predicting estimates of oxygen transmissibility for scleral lenses. Cont Lens Anterior Eye 2012;35(6):266-71.

14. Vincent SJ, Alonso-Caneiro D, and Collins MJ. The temporal dynamics of miniscleral contact lenses: Central corneal clearance and centration. Cont Lens Anterior Eye 2018;41(2):162-68.

15. Courey $\mathrm{C}$ and Michaud L. Variation of clearance considering viscosity of the solution used in the reservoir and following scleral lens wear over time. Cont Lens Anterior Eye 2017;40(4):260-66.

16. Collins MJ, et al, Flexure of thin rigid contact lenses. Cont Lens Anterior Eye 2001;24(2):59-64

17. Phillips AJ and Stone J Contact Lenses Third ed. London, UK: Butterworths; 1989.

18. Sorbara L, Fonn D and MacNeill K. Effect of rigid gas permeable lens flexure on vision. Optom Vis Sci 1992;69(12):953-8.

19. Choi J, et al. Changes of ocular higher order aberration in on- and off-eye of rigid gas permeable contact lenses. Optom Vis Sci, 2007;84(1):42-51.

J Cont Lens Res Sci Vol 5(1):e1-e8; January 14, 2021

This article is distributed under the terms of the Creative Commons Attribution-Non Commercial 4.0 International License. CMichaud and Courey. 
20. Chen M and Yoon G. Posterior corneal aberrations and their compensation effects on anterior corneal aberrations in keratoconic eyes. Invest Ophthalmol Vis Sci 2008;49(12):5645-52.

21. Hashemi $\mathrm{H}$, et al. Pentacam top indices for diagnosing subclinical and definite keratoconus. J Curr Ophthalmol 2016;28(1):21-6.

22. Barnett $M$ and Fadel D. Scleral Lenses: The Benefits of Toric Landing Zones. C.L. Spectrum 2017;32(November):36-41.
23. Sabesan R, et al., Wavefront-guided scleral lens prosthetic device for keratoconus. Optometry and vision science : official publication of the American Academy of Optometry 2013;90(4):314-323.

24. Nave R. Coma. 2019; Available at: http://hyperphysics. phy-astr.gsu.edu/hbase/geoopt/coma.html accessed 2019-10-25

J Cont Lens Res Sci Vol 5(1):e1-e8; January 14, 2021

This article is distributed under the terms of the Creative Commons Attribution-Non

Commercial 4.0 International License. @Michaud and Courey. 